\title{
Mises à jour apportées au Guide canadien d'immunisation: De mars 2014 à mars 2015
}

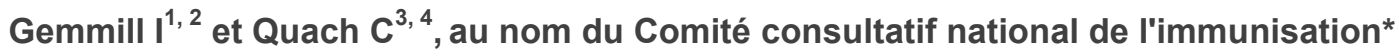

\footnotetext{
${ }^{1}$ Président du Comité consultatif national de l'immunisation

2 Bureau de santé de Kingston, Frontenac, Lennox et Addington, Kingston (Ontario)

${ }^{3}$ Coprésidente du CCNI et du groupe de travail sur les pneumococcies, Montréal (Québec)

${ }^{4}$ Centre d'études sur les vaccins, Centre universitaire de santé McGill, Montréal (Québec)
}

*Correspondance : naci-ccni@phac-aspc.gc.ca

\section{Résumé}

Le Comité consultatif national de l'immunisation (CCNI) élabore des recommandations sur l'utilisation des vaccins pour les Canadiens, qui sont résumées dans le Guide canadien d'immunisation (ci-après " le Guide »), lequel est régulièrement mis à jour. Des recommandations concernant cinq vaccins ont été émises entre mars 2014 et février 2015. Les mises à jour du Guide comprennent des recommandations formulées au sujet de l'administration d'un autre dosage du vaccin contre le virus du papillome humain (VPH) chez les adolescents, du moment exact choisi pour l'administration de l'immunoglobuline antivaricelleuse (Varlg) suivant une exposition à la varicelle, et des vaccins contre le méningocoque et vaccins quadrivalents antigrippaux dont l'utilisation a récemment été autorisée au Canada. Un changement a également été apporté aux recommandations concernant l'utilisation des vaccins contre le pneumocoque chez les adultes et les personnes atteintes d'asthme. Le chapitre portant sur le vaccin contre l'encéphalite transmise par les tiques a été retiré du Guide, car ce vaccin n'est plus offert au Canada.

\section{Introduction}

Le Comité consultatif national de l'immunisation ( $\mathrm{CCNI}$ ) émet des recommandations concernant l'utilisation des vaccins pour les Canadiens. II constitue l'organisme scientifique consultatif de l'Agence de la santé publique du Canada (ASPC) sur l'immunisation (1). Ces recommandations, ainsi que d'autres renseignements sur l'immunisation, sont publiées dans le Guide canadien d'immunisation (ci-après « le Guide ») (2).

Depuis sa première édition en 1979 , le Guide constitue un résumé fiable et facile à lire utilisé par les professionnels de la santé pour donner des conseils à leurs patients, et par les responsables des politiques pour exécuter les programmes d'immunisation. Le document comporte cinq parties, qui traitent des renseignements clés sur l'immunisation, de l'innocuité des vaccins, des populations particulières, des vaccins actifs et des agents d'immunisation passive. Depuis l'édition de 2006, le Guide a subi d'importantes révisions. II est depuis 2012 publié en ligne dans un format électronique évolutif (2). L'objectif de cet article consiste à mettre en évidence les mises à jour apportées au Guide entre le $1^{\text {er }}$ mars 2014 et le 31 mars 2015.

\section{Approche}

Durant l'élaboration de ses déclarations, le CCNI effectue des synthèses approfondies de connaissances et des analyses englobant des examens scientifiques, des pratiques en évolution et des recommandations à l'échelle nationale et internationale. Le CCNI transmet ses recommandations sous forme résumée dans les chapitres correspondants du Guide. Des recommandations détaillées concernant l'immunisation et l'utilisation des vaccins offerts au Canada se trouvent dans les déclarations et les mises à jour des déclarations du CCNI, qui sont disponibles sur le site Web de l'ASPC (1). 
Résumé des mises à jour

Le Tableau 1 offre un résumé des récents changements et ajouts apportés au Guide.

Tableau 1 : Faits saillants des principaux changements apportés aux recommandations concernant les vaccins actifs dans le Guide canadien d'immunisation de mars 2014 à mars 2015

\begin{tabular}{|c|c|}
\hline \multirow[t]{2}{*}{ Virus du papillome humain } & $\begin{array}{l}\text { Le CCNI recommande désormais pour les adolescents de } 9 \text { à } 14 \text { ans immunocompétents } \\
\text { et non infectés par le VIH un calendrier de deux doses (vaccin VPH2 ou VPH4 à } 0 \text { mois } \\
\text { ou entre } 6 \text { et } 12 \text { mois) ou de trois doses (vaccin VPH2 à } 0,1 \text { et } 6 \text { mois ou vaccin VPH4 à } \\
0,2 \text { et } 6 \text { mois). }\end{array}$ \\
\hline & $\begin{array}{l}\text { On recommande l'administration de trois doses de vaccin contre le VPH (vaccin VPH4 } \\
\text { pour les garçons et les filles à } 0,2 \text { et } 6 \text { mois ou vaccin VPH2 pour les filles à } 0,1 \text { et } \\
6 \text { mois) pour les personnes immunodéprimées et immunocompétentes infectées par le } \\
\text { VIH. }\end{array}$ \\
\hline \multirow[t]{3}{*}{ Grippe } & $\begin{array}{l}\text { Deux vaccins antigrippaux inactivés quadrivalents (VAQ) ont été autorisés au Canada } \\
\text { (Flulaval }^{\mathrm{MC}} \text { Tetra et Fluzone }{ }^{\mathrm{MD}} \text { Quadrivalent). }\end{array}$ \\
\hline & $\begin{array}{l}\text { Les personnes allergiques aux œufs peuvent se faire vacciner contre la grippe dans tout } \\
\text { lieu où se déroule habituellement la vaccination et y recevoir une dose complète du } \\
\text { vaccin trivalent inactivé (VTI) ou du vaccin VAQ sans subir au préalable de test cutané. }\end{array}$ \\
\hline & $\begin{array}{l}\text { Le CCNI recommande l'utilisation préférentielle, lorsque cela est possible, du vaccin } \\
\text { antigrippal vivant atténué (VVAI) pour les enfants de moins de } 6 \text { ans. Le CCNI se fonde } \\
\text { sur des preuves de l'efficacité supérieure du VVAI par rapport à celle du VIT pour ces } \\
\text { enfants, ce qui n'a pas été démontré aussi clairement chez les enfants plus âgés. Bien } \\
\text { que l'on s'attende à ce que l'efficacité supérieure du VVAI par rapport à celle du VTI se } \\
\text { prolonge après l'âge de } 6 \text { ans, il n'y a présentement pas de données indiquent à quel âge } \\
\text { précis l'efficacité des deux vaccins devient équivalente. Si le VVAI n'est pas disponible } \\
\text { pour les personnes pour lesquelles il est recommandé, le VTI devrait être utilisé. }\end{array}$ \\
\hline \multirow[t]{3}{*}{ Méningocoque } & $\begin{array}{l}\text { Deux nouveaux vaccins inactivés contre le méningocoque ont été homologués au } \\
\text { Canada : le vaccin multicomposant contre le méningocoque du sérogroupe } B(4 C M e n B \text {, } \\
\text { Bexsero }{ }^{\mathrm{MC}} \text { ) et le vaccin conjugué contre le méningocoque, sérogroupes } A, C, Y \text { et } W-135 \\
\left(\text { Nimenrix }^{\mathrm{MC}}\right) \text {. }\end{array}$ \\
\hline & $\begin{array}{l}\text { Le CCNI recommande l'administration du vaccin 4CMenB pour les personnes de } 2 \text { mois } \\
\text { et plus qui possèdent les caractéristiques suivantes : } \\
\text { - } \quad \text { elles présentent un risque élevé de contracter une méningococcie invasive (MI) } \\
\text { attribuable au Neisseria meningitidis du sérogroupe B; } \\
\text { - } \quad \text { elles se sont trouvées en contact étroit avec un cas de méningococcie invasive } \\
\text { attribuable au Neisseria meningitidis du sérogroupe B; } \\
\text { - elles se révèlent à risque pendant les éclosions de méningococcie invasive } \\
\text { attribuable au Neisseria meningitidis du sérogroupe B. }\end{array}$ \\
\hline & $\begin{array}{l}\text { N'importe lequel des vaccins conjugués quadrivalents }(\mathrm{A}, \mathrm{C}, \mathrm{Y} \text { et } \mathrm{W}-135) \text { peut être utilisé } \\
\text { pour les personnes à risque élevé âgées de } 2 \text { ans et plus ou pour les voyageurs âgés de } \\
2 \text { ans et plus qui se rendent dans des régions où le vaccin contre le méningocoque est } \\
\text { recommandé. }\end{array}$ \\
\hline \multirow[t]{2}{*}{ Pneumocoque } & $\begin{array}{l}\text { Le CCNI recommande la vaccination au moyen du vaccin contre le pneumocoque indiqué } \\
\text { selon l'âge aux personnes ayant eu recours à des soins médicaux pour l'asthme au cours } \\
\text { des } 12 \text { mois précédents. }\end{array}$ \\
\hline & $\begin{array}{l}\text { Les personnes présentant des problèmes médicaux sous-jacents qui leur font courir un } \\
\text { risque élevé de pneumococcie invasive devraient recevoir une dose de rappel unique du } \\
\text { vaccin Pneu-P-23 } 5 \text { ans après la première dose, quel que soit leur âge à la première } \\
\text { dose. }\end{array}$ \\
\hline
\end{tabular}




\begin{tabular}{|c|c|}
\hline & $\begin{array}{l}\text { Une dose de vaccin polysaccharidique contre le pneumocoque (Pneu-P-23) est } \\
\text { recommandée pour tous les adultes de } 65 \text { ans et plus dès lors que } 5 \text { années se sont } \\
\text { écoulées depuis la dernière dose de Pneu-P-23, et pour les adultes immunocompétents } \\
\text { de moins de } 65 \text { ans qui vivent dans un établissement de soins de longue durée ou dont } \\
\text { l'état de santé ou la situation a pour effet d'augmenter leur risque de contracter une } \\
\text { pneumococcie. }\end{array}$ \\
\hline & $\begin{array}{l}\text { Toutes les personnes qui ont déjà reçu le vaccin Pneu-P-23 et qui ont besoin d'être } \\
\text { vaccinées à nouveau avec le vaccin conjugué contre le pneumocoque (Pneu-C-13) } \\
\text { devraient recevoir le vaccin Pneu-C-13 au plus tôt cinq ans après la dernière dose de } \\
\text { Pneu-P-23. }\end{array}$ \\
\hline & $\begin{array}{l}\text { Le vaccin Pneu-C-13 devrait être administré aux adultes atteints d'affections liées à } \\
\text { l'immunodépression, suivi du vaccin Pneu-P-23 au moins huit semaines plus tard - s'il } \\
\text { n'a pas déjà été administré. }\end{array}$ \\
\hline $\begin{array}{l}\text { Encéphalite transmise par } \\
\text { les tiques }\end{array}$ & Le vaccin contre l'encéphalite transmise par les tiques n'est plus offert au Canada. \\
\hline Varicelle & $\begin{array}{l}\text { Afin d'assurer le maximum de protection, l'immunoglobuline antivaricelleuse devrait être } \\
\text { administrée dès que possible suivant l'exposition, idéalement dans les } 96 \text { heures suivant } \\
\text { la première exposition, mais elle peut être administrée jusqu'à } 10 \text { jours après la dernière } \\
\text { exposition. }\end{array}$ \\
\hline
\end{tabular}

\section{Conclusion}

Depuis 2012, le Guide a été continuellement mis à jour afin d'y intégrer les nouvelles données scientifiques et les nouvelles pratiques tel que le reflètent les déclarations et les mises à jour des déclarations du CCNI les plus récentes. Le CCNI et l'Agence de la santé publique du Canada s'engagent à fournir des renseignements concernant l'immunisation et les vaccins offerts au Canada dans un format facilement accessible et lisible grâce à des mises à jour opportunes et suivies du Guide.

\section{Remerciements}

Les auteurs tiennent à remercier les membres extrêmement dévoués du Comité consultatif national de l'immunisation et le personnel de l'Agence de la santé publique du Canada qui soutiennent le processus de mise à jour du Guide canadien d'immunisation.

\section{Conflit d'intérêts}

\section{Aucun}

\section{Références}

(1) National Advisory Committee on Immunization. NACI Statements, Recommandations and Updates. 2015. http://www.phac-aspc.gc.ca/naci-ccni disponible en français: http://www.phac-aspc.gc.ca/naci-ccni/index-fra.php).

(2) National Advisory Committee on Immunization. Canadian Immunization Guide. 2015. http://www.phac-aspc.gc.ca/publicat/cig-gci/ (disponible en français: http://www.phac-aspc.gc.ca/publicat/cig-gci/index-fra.php). 


\section{Actualités sur les maladies infectieuses : La récente éclosion de rougeole}

Agence de la santé publique du Canada. Rapport hebdomadaire de surveillance de la rougeole et de la rubéole. Semaine 13 de 2015 : du 29 mars au 4 avril 2015.

http://www.phac-aspc.gc.ca/mrwr-rhrr/2015/w08/index-fra.php

- Au Canada, trois nouveaux cas de rougeole ont été signalés par la Colombie-Britannique au cours de la semaine 13.

- Deux provinces (le Québec et la Colombie-Britannique) ont signalé une activité récente de la rougeole. Ces événements ne sont pas liés, car les génotypes sont différents et ils proviennent d'importations distinctes (B3 des États-Unis et $\mathrm{H} 1$ de la Chine, respectivement).

- Au total, 183 cas de rougeole ont été signalés au Canada en 2015.

Figure 1 : Nombre de cas de rougeole $(n=183)$ par semaine d'apparition de l'éruption cutanée, conformément aux déclarations transmises au Système canadien de surveillance de la rougeole et de la rubéole (SCSRR) et au système de surveillance de la rougeole et de la rubéole (MARS), pour la période se terminant le 4 avril 2015.

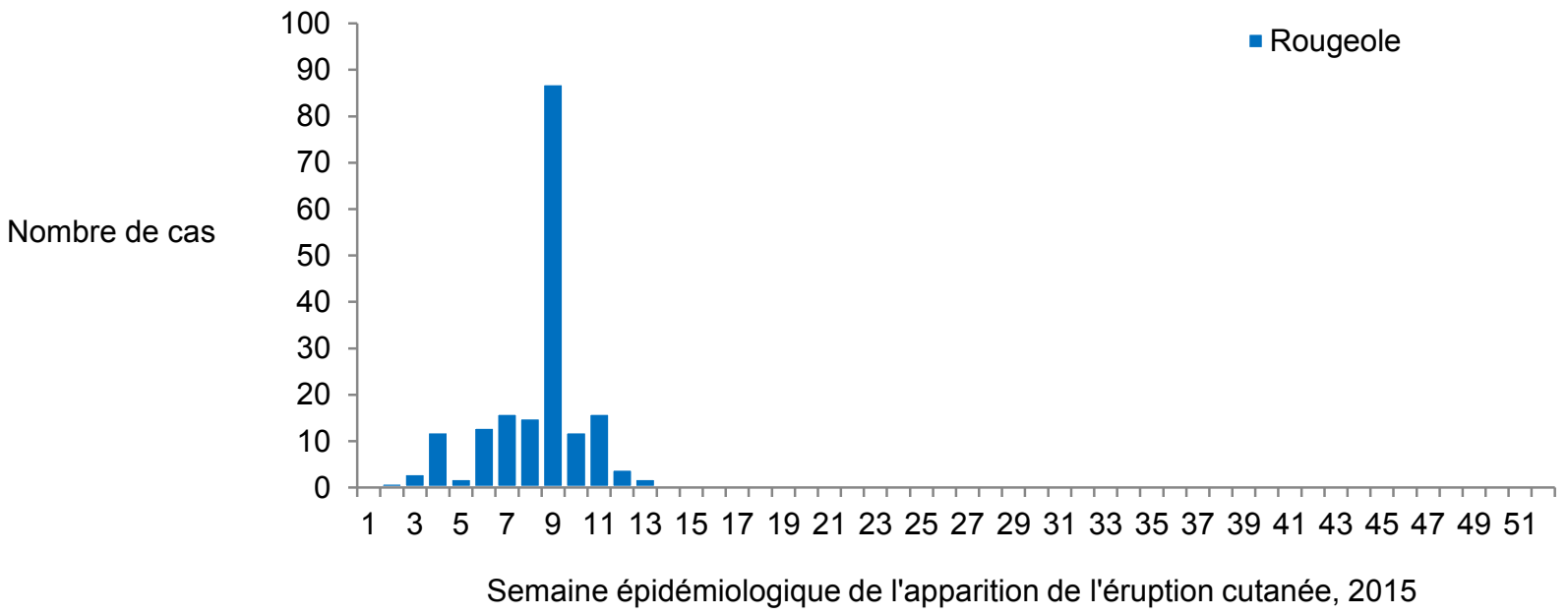

Zipprich J, Winter K, Hacker J, Xia D, Watt J, Harriman K. Measles outbreak-California, December 2014February 2015. Morbidity and Mortality Weekly Report (MMWR). 2015 Feb 20;64(06):153-4 (pasdisponible en français).

http://www.cdc.gov/mmwr/preview/mmwrhtml/mm6406a5.htm?s_cid=mm6406a5_w

Le 5 janvier 2015, le California Department of Public Health a été informé d'un cas suspect de rougeole. En date du 11 février, un total de 125 cas de rougeole avec une éruption cutanée s'étant produite au cours de la période du 28 décembre 2014 au 8 février 2015 avaient été confirmés parmi les résidents des États-Unis concernés par cette éclosion. Parmi les 125 cas, 110 patients résidaient en Californie. Trente-neuf (35\%) des patients de Californie se sont rendus dans l'un ou dans les deux parcs thématiques de Disney durant la période du 17 au 20 décembre, où ils semblent avoir été exposés au virus de la rougeole. Parmi les 110 patients de Californie, 49 (45\%) n'étaient pas vaccinés, cinq $(5 \%)$ avaient reçu une dose du vaccin contenant le virus de la 
rougeole, sept (7) avait reçu deux doses, un (1\%) avait reçu trois doses, et 47 (43\%) avaient un statut vaccinal inconnu ou non documenté.

La présence annuelle au parc thématique Disney en Californie est estimée à 24 millions, incluant des visiteurs internationaux provenant de pays où la rougeole est endémique. Cette épidémie démontre l'importance d'assurer une couverture maximale contre la rougeole aux États-Unis.

\section{Invitation à participer au sondage du CCNI}

Nous faisons appel à votre participation à un important sondage lancé par l'Agence de la santé publique du Canada (l'Agence) dans le but d'évaluer les opinions et les suggestions d'intervenants clés en matière d'immunisation sur les ressources élaborées par le Comité consultatif national de l'immunisation (CCNI).

II est très important que les conseils et les produits d'information du CCNI répondent aux besoins de ceux qui fournissent des vaccins, qui élaborent des recommandations en matière de politiques sur les vaccins ou qui travaillent autrement dans le domaine des vaccins ou de l'immunisation. Les lecteurs du RMTC ont été identifiés en tant que partenaires du CCNI. Nous vous prions de bien vouloir nous fournir des commentaires qui permettront d'améliorer les produits du CCNI liés aux recommandations en matière d'immunisation par l'entremise d'un sondage qui prendra environ dix minutes. Vos commentaires sont essentiels pour assurer la satisfaction de vos besoins comme intervenant. Veuillez profiter de cette occasion pour vous faire entendre : cliquez sur le lien ci-dessous afin de répondre au sondage avant le 12 mai 2015 . Vos réponses seront entièrement confidentielles; aucune personne ne sera identifiée de quelque façon que ce soit. Pour toute question, veuillez envoyer un courriel à l'adresse naci-ccni@phac-aspc.gc.ca.

Vous pouvez accéder au sondage à partir du lien ci-après : http://surveys-sondages.hc-sc.gc.ca/s/NAClCCNI/langfral

Nous vous remercions de votre soutien! 